\title{
Design of unmanned patrol ship for marine culture based on STM32
}

\author{
Chen Yu-jie ${ }^{1, a}$, Liu Pei-xue ${ }^{1, b}$, Feng Fei ${ }^{1, c}$, Chen Hui-wei ${ }^{1, d}$ \\ Qingdao Huanghai College, Qingdao 266427, China \\ achenyujie_cc@163.com, b93987898@qq.com \\ c1547752800@qq.com, d83430761@qq.com
}

\begin{abstract}
Based on STM32F103C8T6 SCM unmanned cruise ship support rocker control and GPS navigation two modes. Through the GPS navigation system to achieve automatic cruise, you can automatically avoid obstacles, and real-time transmission of its own positioning information to the monitoring end. Unmanned ship monitoring terminal receiving arrangement through Bluetooth tasks and route selection, arrive at the mariculture area task, water quality testing operations, and the detection of water quality data sent via Bluetooth to the monitoring terminal for processing and display. The research of this topic not only has important theoretical value, but also has a very broad application prospect.
\end{abstract}

\section{Keywords: Unmanned boat; Rocker operation; Autonomous cruise}

\section{Introduction}

Unmanned inspection ship is a kind of industrial robot. Its working environment is the sea surface. It can realize the functions of autonomous cruise and water quality detection. In recent years, the sea industry has paid more and more attention to it. With the development of aquaculture, computer technology, sensor technology and network technology, unmanned inspection ship is developing rapidly.

Considering the complex nature of the marine environment, marine aquaculture environment unmanned patrol boats is different from the general robot, it can meet the needs of the general characteristics of the intelligent control of the robot, it should be according to the environmental characteristics of their application. To sum up, the design of unmanned inspection ship based on embedded STM32 not only has very important theoretical value, but also has a very broad application prospect. The unmanned patrol ship is small in size and high in maneuverability. It can be used for route planning through maps, and guide unmanned patrol boats to the designated destination to monitor.

\section{Overall System Design}

In the rocker mode, the unmanned ship is controlled to the target point to monitor the water quality. GPS positioning mode, operators in the upper computer's map, set the end of the unmanned ship and the task of operation, in the process of driving, rely on the GPS navigation system. In this process, the real-time transmission of its location information to a computer terminal to realize map positioning function, water quality monitoring to arrive after using the collection module, water quality monitoring using PH sensor, the temperature sensor to test the water suitability of fish, display in the computer; in the control system, using the closed loop control mode, unmanned boat received PWM wave to control the output of different PWM were different, PWM wave output is used to adjust the speed and direction of the unmanned boat, thus ensuring unmanned ships can travel to constantly adjust end point, after finally reaching the end point. At the same time, GPS data module main control chip using a serial interface to read the unmanned boat, through a simple data processing will be carried out after the communication data of unmanned ship longitude, 
seawater $\mathrm{pH}$, and temperature through the first serial port and Bluetooth, then through Bluetooth network can transmit data to the host computer, the host computer receives the data after the data conversion work, marking unmanned ship position and now have been driving over the track on the map.

During the operation, no ship may face water obstacle problems, so the six infrared photoelectric sensor to judge whether there are obstacles in front of the waterway condition, if encounter obstacles around the hull itself obstacles, and then accept the path data of PC again, by running operation according to the instruction content.

\section{Hardware Design}

\section{Hull section}

The model specification of unmanned ship in design is length $40 \mathrm{~cm}$, width $22 \mathrm{~cm}$, and the speed of unmanned vessel is about $1 \mathrm{~m} / \mathrm{s}$. No one in the boat, mainly using PWM wave to control the stern rudder angle, which can be used to determine the direction of travel of the hull. The angle of the stern is 90 degrees, including the distance between 45 degrees on the left and 45 degrees on the right. The system uses $7.4 \mathrm{~V}$ model as the ship model lithium battery power supply, the maximum output current of 2A, capacity of $800 \mathrm{mAh}$, and then through the AMS1117 conversion module for $5 \mathrm{~V}$ and $3.3 \mathrm{~V}$ variable voltage is the main control module and the power supply of each sensor. The equipment needed for the power supply system is respectively three-phase brushless motor, propeller and infrared photoelectric sensor module.

In the direction of the ship's conversion, the stern swing is used to support the GPS control, which can be converted to the desired angle and maintained at this angle. In this system, the unmanned ship uses the digital steering gear to control the stern. Its torque is big, the reaction speed is fast, and the control precision is accurate.

\section{Control section}

The unmanned ship will STM32F103C8T6 as the main control chip of the system, which can provide a stable PWM wave for external equipment, mainly used to control the DC motor speed and the steering angle of rotation, the feedback signal and is also responsible for monitoring the wireless signal module to calculate the distance, the single chip is responsible for receiving GPS data module information.

STM32F103C8T6 is an integrated circuit, with 32 bit microcontroller, belonging to medium capacity enhancement type, power consumption is very low. It has high instruction set and single cycle instruction execution time. STM32F103C8T6 can coordinate the speed of its processing and the power consumption of the system very well. The efficiency of the STM32F103C8T6 execution code is very high because the structure of its internal core is Harvard, which is characterized by the direct connection of the register to the arithmetic logic unit.

\section{Transmission section}

In the data transmission of unmanned ships, Bluetooth is the main mode of transmission. All the Bluetooth HC-05 Bluetooth module, the output voltage of about $3.6 \mathrm{~V}$ to $6 \mathrm{~V}$, the baud rate is set by user, and embedded LED, if flash said no Bluetooth connection, if slow flash has entered the AT command mode. If it is divided according to the automatic connection mode, it is divided into the main circuit, the slave circuit and the three working states. When the Bluetooth module works automatically, the Bluetooth will automatically connect in accordance with the preset way and transfer the data. When the module is in the mode that commands work accordingly, all the settings for AT will be executed.

In the unmanned marine environment monitoring system, the GPS-NEO-7M-C module is mainly used to provide the latitude and longitude information of the location of an unmanned ship during the process of traveling, so as to complete the map positioning function of the computer terminal. The current GPS module, its GPS chip is mostly selected SiRFIII series based. The GPS module 
has the active antenna with high gain, but also support the $3.3 \mathrm{~V}$ and $5 \mathrm{~V}$ power supply, the baud rate to support the common 9600, and with IPX interface to increase the gain range of active antenna, the positioning accuracy of $2.5 \mathrm{mCEP}$, can fully meet the real-time positioning of unmanned ship position requirements. In general, the sensitivity is high, thereby shortening the positioning time and rapidly entering the positioning application state. This module is shown in figure 1.

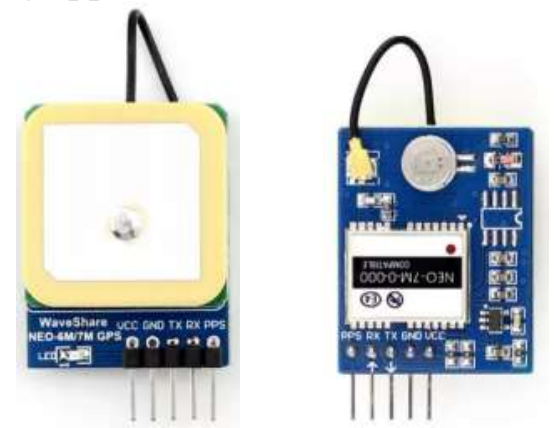

Figure 1 GPS positioning module physical map

\section{Software Design}

\section{Main program}

After completion of the initialization operation, the system master function enters the loop waiting for data monitoring results or interrupt signals. In the process of unmanned boat travel, may encounter various emergency situations, in order to realize the obstacle avoidance, this system with six infrared photoelectric sensor scans, used to determine the front and around the obstacle range, steering control unmanned boat finally according to the result of the judgment. According to the situation detected by the six infrared photoelectric sensor modules, the unmanned ship is operated in the mode of self cruising, and the interruption of the serial port of the shielding and Bluetooth nodes is.

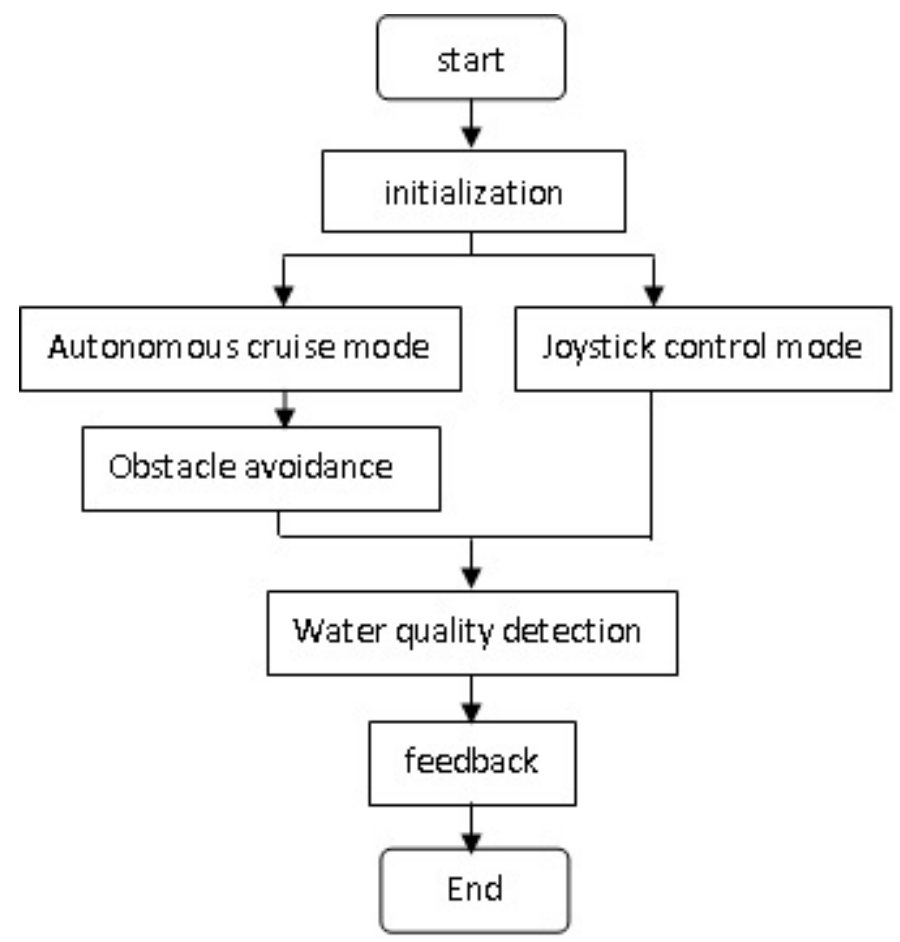

Figure 2 flow chart of main program 


\section{Bluetooth transmit serial data}

The Bluetooth module connected with the computer is set up as coordinator to build the network, and the GPS module on the unmanned ship is configured as routing node. In the design, the ways of information transmission on Bluetooth, the main work process: the transmission mode between unmanned boat and PC terminal for Bluetooth, unmanned boat using Bluetooth to receive control instructions from the client computer, and then according to the contents of corresponding instruction. On the other hand, the unmanned ship also uses Bluetooth to transmit the collected data to the computer terminal for processing and display.

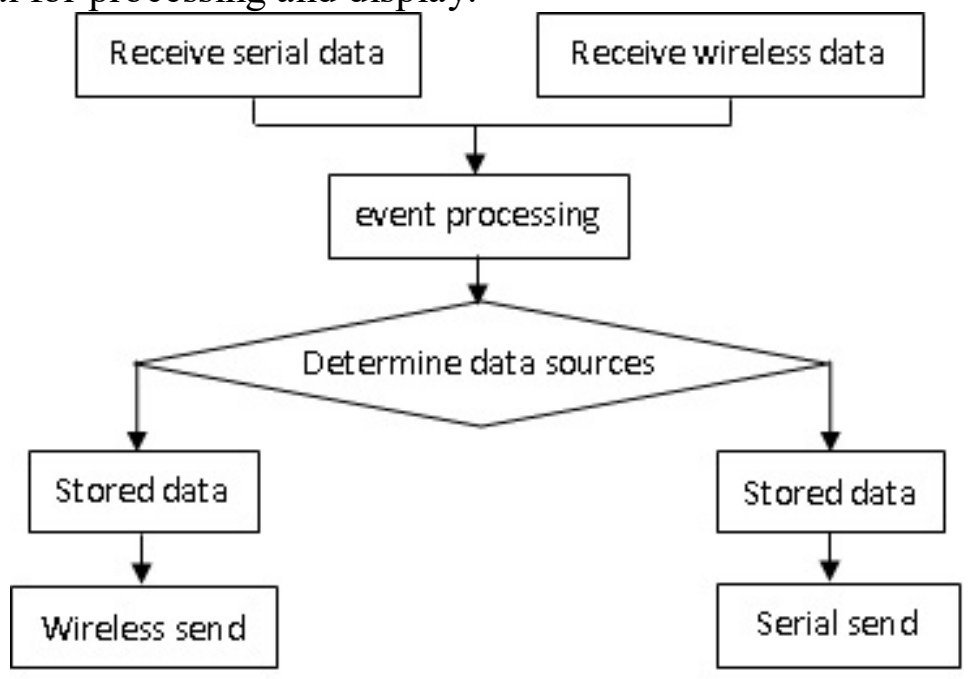

Figure 3 flow chart of data transmission

\section{System Test}

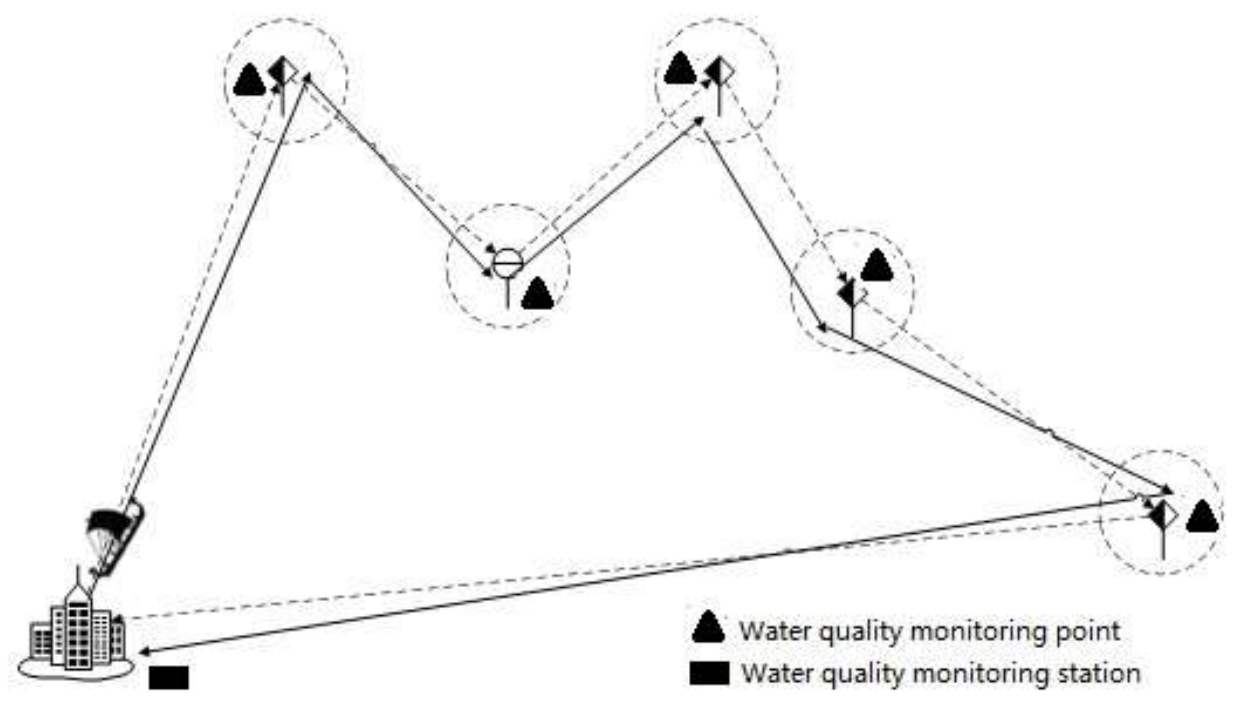

Figure 4 schematic diagram of cruise condition of unmanned ship

In the design, the unmanned ship adopts the GPS navigation mode. Because the water quality monitoring point is in the water, the unmanned ship can read data and complete the preset task of the system only by entering the effective distance of the detection point. In addition, the unmanned ship in addition to relying on GPS navigation, as well as remote control of the coordination. When the unmanned vessel performs reading data tasks, the navigation status of its path is shown in figure 4. The dotted circle around each node is the effective transmission range of the water quality monitoring point, the dotted line is the map planning line, and the solid line part is the real cruise route of the unmanned ship. 
According to the above situation, autonomous cruise mode test can be converted to unmanned ships during cruise in the task arrangement required number of nodes collecting data, node data collection of actual unmanned ship correctly, the test results are shown in table 1.

Table 1 test data recording chart for cruise process

\begin{tabular}{rcc}
\hline $\begin{array}{c}\text { Number of } \\
\text { task nodes }\end{array}$ & $\begin{array}{c}\text { Number of actual } \\
\text { nodes }\end{array}$ & $\begin{array}{c}\text { Probability of losing } \\
\text { points }\end{array}$ \\
\hline 10 & 9 & $10 \%$ \\
13 & 13 & $0 \%$ \\
16 & 16 & $0 \%$ \\
19 & 19 & $0 \%$ \\
20 & 19 & $5 \%$ \\
\hline
\end{tabular}

\section{Conclusion}

Aiming at the current water quality monitoring problem, this paper designs and develops an unmanned cruise ship based on embedded system. After the prototype development and testing, the unmanned ship basically achieved the desired results. The operator task and work on the computer side route choice, and then through the Bluetooth data sent to the unmanned boat, unmanned boat on GPS navigation, in this process, if you encounter obstacles, can avoid obstacles, and the GPS data's real-time transmission to the computer terminal to achieve positioning map. When the unmanned ship arrives at the mission point, the water quality monitoring task is carried out, and the test data is transmitted to the computer end through Bluetooth to calculate and reality. In order to improve the control mode of unmanned ship, the remote control is added.

\section{Acknowledgement}

The project number: 2017GGX201004; 2016kj04; J16LN78

\section{References}

[1] Sun Juan, Yang Deli. Study on sustainable development of mariculture in China [J]. Shanxi agricultural science, 2014,39 (7): 733-735746.

[2] Zhang Jie. Design and implementation of a safety inspection system for marine aquaculture based on wireless networks [J]. Journal of Hebei Academy of Sciences, 2013,12 (3): 5-27.

[3] Zhang Lanpeng. Design of information module for unmanned ship based on information fusion technology [D]. Wuhan: University of science and engineering, 2015. [4] Zhang Guoqian. Design of unmanned ship motion system based on ZigBee remote wetland monitoring system [D]. Hebei: Hebei University, 2014.

[5] Chen Yujie, Liu Peixue, Gao Ying. Design of water quality monitoring system for sea cucumber culture based on ZigBee network [J]. modern electronic technology, 2016,39 (23): 100-103. 